\title{
VASSILIEV KNOT INVARIANTS AND LIE $S$-ALGEBRAS
}

\author{
ARKADY VAIntrob
}

\begin{abstract}
The goal of this work is to explain the appearance of Lie algebras in the theory of knot invariants of finite order (Vassiliev invariants). As a byproduct, we find a new construction of such invariants. Namely, we show that the theory of Vassiliev invariants leads naturally to the notion of $S$-Lie algebra, where $S$ is an involutive solution of the quantum Yang-Baxter equation. For each $S$-Lie algebra $L$ with an $L$-invariant $S$ symmetric non-degenerate bilinear form $b$ and an invariant functional on its universal enveloping algebra, we construct a sequence of Vassiliev knot invariants.
\end{abstract}

\section{Introduction}

The Jones polynomial and its generalizations were constructed by combinatorial methods motivated by statistical physics. Witten in [15] gave a heuristic path integral interpretation of these invariants. Bar-Natan [2] found that perturbative approach to Witten's integrals is related to Vassiliev's theory of knot invariants of finite type. Therefore, there is an indirect connection between models of statistical physics and Vassiliev invariants. In this paper we will give a direct combinatorial construction of Vassiliev invariants. The idea is to adopt to the case of Vassiliev diagrams the Yang-Baxter models for the Jones polynomial and its relatives $[5,10]$.

The works of Birman-Lin [1], Bar-Natan [2, 3] and Kontsevich [5] show that Vassiliev invariants (with values in $\mathbf{Q}$ ) can be described by combinatorial objects called weight systems. Weight systems of order $n$ form a finite-dimensional vector space $W_{n}$. To describe this space, we just need to solve a system of linear equations. But the numbers of unknowns and equations in this system grow extremely fast. For example, to find that $\operatorname{dim} W_{9}=44$, Bar-Natan [2] had to work with a linear system of 5,056,798 equations and 644,808 unknowns, and it took 10 days of computer time. There is absolutely no hope of computing $\operatorname{dim} W_{10}$ using this approach. Taking into account a relatively small number of independent solutions for a system of so huge a size, we need to look for a better way to describe $W_{n}$.

Bar-Natan in [2] gave a construction of weight systems using a Lie algebra $L$ with an invariant inner product and a module. These invariants

Received April 27, 1994.. 
of finite type appear as coefficients of the perturbative expansion of the corresponding Chern-Simons-Witten invariant.

The appearance of Lie algebras in the knot-theoretic situation looks slightly mysterious since there seem to be no direct links between knots and Lie algebras. We will discuss such relation here.

Specifically, we take the idea of operator-valued invariants of knots and links (see $[11,16]$, for example) and apply it to the case of weight systems and chord diagrams. We show that this path naturally leads to the notion of Yang-Baxter Lie algebra. These algebraic structures generalize both Lie algebras and Lie superalgebras. They appeared earlier in the literature on the quantum Yang-Baxter equation and quantum groups (cf. $[4,6]$ ), but only in the language of chord and Feynman diagrams do they obtain a natural pictorial interpretation. As a byproduct, we will find a more general construction of weight systems. Our main result can be formulated as follows.

Let $S$ be an involutive solution of the quantum Yang-Baxter equation. For each $S$-Lie algebra $L$ with an $L$-invariant $S$-symmetric non-degenerate bilinear form $b$ on $L$ and an invariant functional on its universal enveloping algebra, we construct a sequence of Vassiliev knot invariants.

Bar-Natan conjectured that the space of Vassiliev invariants is spanned by the invariants coming from Lie algebras. If confirmed, this conjecture would contradict Vassiliev's completeness conjecture that invariants of finite order are able to distinguish all different knot types.

Our construction is a priori more general than Bar-Natan's. It has not yet brought us new invariants. However, even if Bar-Natan's conjecture is true, many interesting knot invariants will appear only as linear combinations of invariants coming from several Lie algebras and modules. A typical example is the Alexander-Conway polynomial $\Delta(z)=a_{0}+a_{1} z+a_{2} z^{2}+\ldots$. Each coefficient $a_{n}$ is a knot invariant of finite order, but no Lie algebra can produce the whole sequence $a_{0}, a_{1}, \ldots, a_{n}, \ldots$ by Bar-Natan's construction. This can be achieved if we use weight systems coming from Yang-Baxter Lie algebras.

\section{Vassiliev invariants}

Here we summarize results of Vassiliev [13, 14], Birman-Lin [1], Kontsevich [5], and Bar-Natan [3] on Vassiliev knot invariants.

1.1. Definition of Vassiliev invariants. Denote by $\mathcal{K}$ the space of all oriented knots in $\mathbb{R}^{3}$. By $\overline{\mathcal{K}}$ we denote the space of immersions of $S^{1}$ into $\mathbb{R}^{3}$, the only possible singularities of which are simple double points (i.e. self-intersections with distinct tangents). Elements of $\overline{\mathcal{K}}$ are called singular knots. Let $\mathcal{K}_{p}$ be the subspace of $\overline{\mathcal{K}}$ consisting of singular knots with not more than $p$ self-intersections. It is clear that $\overline{\mathcal{K}}=\bigcup_{p} \mathcal{K}_{p}$ and $\mathcal{K}_{0}=\mathcal{K}$. 
The starting point of Vassiliev's theory is a simple observation that any numerical knot invariant can be naturally extended to the set of singular knots.

Proposition 1.1.1. Let $I: \mathcal{K} \rightarrow k$ be a knot invariant with values in an Abelian group $k$. There exists an extension of $I$ to $\overline{\mathcal{K}}$ such that

(1)

$$
I\left(K_{+}\right)-I\left(K_{-}\right)=I\left(K_{0}\right),
$$

where $K_{+}, K_{-}$and $K_{0}$ are three singular knots identical everywhere except the interior of a small ball in $\mathbb{R}^{3}$ where they look like

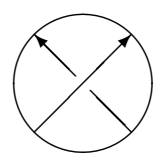

$K_{+}$

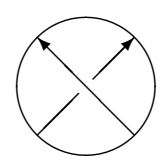

$K_{-}$

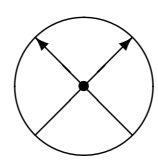

$K_{0}$

$(2)$

$$
I(K)=\sum_{\alpha \in\left(\mathbb{Z}_{2}\right)^{p}} \operatorname{sgn}(\alpha) I\left(K_{\alpha}\right),
$$

where for $\alpha=\left(\alpha_{1}, \ldots, \alpha_{p}\right), \alpha_{i} \in \mathbb{Z}_{2}=\{ \pm 1\}$, we denote by $\operatorname{sgn}(\alpha)$ the product $\prod \alpha_{i}$ and by $K_{\alpha}$ the knot obtained from a singular knot $K \in \mathcal{K}_{p} \backslash \mathcal{K}_{p-1}$ with exactly $p$ double points $x_{1}, \ldots, x_{p}$ by resolving the point $x_{i}$ in a positive (resp. negative) direction if $\alpha_{i}=+1$ (resp. $\left.\alpha_{i}=-1\right)$.

\section{Corollary 1.1.2. [1]}

(1) Value of the extended invariant on a singular knot like

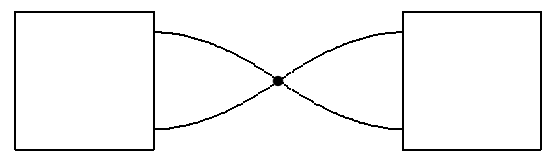

is equal to zero. 
(2) The extension of a knot invariant I satisfies the so-called four-term relation

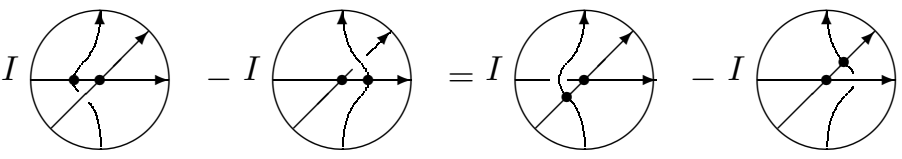

Definition 1.1.3. An oriented knot invariant $I$ is called an invariant of order (less than or equal to) $p$ if its extension to $\overline{\mathcal{K}}$ vanishes on all singular knots with more than $p$ double points, i.e. $I(K)=0$ for $K \in \mathcal{K}_{p+1}$. Invariants of finite order are called Vassiliev invariants.

Denote by $V_{p}$ the space of invariants of order $\leq p$, and by $\mathcal{V}$ the space of all Vassiliev invariants. We have a filtration

$$
V_{0} \subset V_{1} \subset V_{2} \subset \ldots \subset V_{p} \subset V_{p+1} \subset \ldots \subset \mathcal{V} .
$$

If $k$ is a ring, it is easy to see that $V_{p} \cdot V_{q} \subset V_{p+q}$, i.e. $\mathcal{V}$ is a filtered algebra.

All known knot polynomials are related to Vassiliev invariants. For example, let $\Delta(K)=c_{0}+c_{1} z+c_{2} z^{2}+\ldots$ be the Alexander-Conway polynomial of the knot $K$. Then it is easy to see [3] that the coefficient $c_{n}$ is a Vassiliev invariant of order $\leq n$. Birman and Lin [1] showed that after a change of variable in the Jones (or HOMFLY, or Kauffman) polynomial it becomes a formal power series whose $n$-th coefficient is a Vassiliev invariant of order $\leq n$.

Similarly, if $R$ is a deformation of the trivial solution of the quantum Yang-Baxter equation, then the $R$-matrix invariants of Turaev [10] correspond to sequences of Vassiliev invariants (cf. [3]).

1.1.4. Triple intersections. Triple intersections are not allowed in singular knots, but we may use 1.1.2.(2) to show that each invariant $I$ can be extended to the set of immersions $S^{1} \rightarrow \mathbb{R}^{3}$ with transversal double and triple intersections by means of the following three-term relation

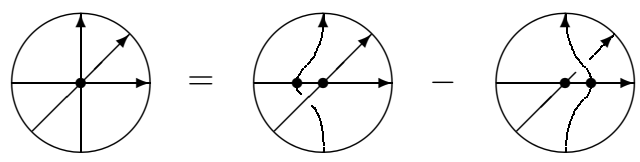

This relation obviously implies the four-term relation 1.1.2.(2). （Here and in the drawings that follow, we omit $I$ in front of diagrams.)

Remark 1.1.5. Vassiliev defined his invariants in terms of a natural stratification of the discriminant in the space of maps $\mathbb{R}^{1} \rightarrow \mathbb{R}^{3}$. The axiomatic approach we are using here belongs to Birman and Lin [1]. It allows us to extend the whole theory to invariants of links, and also of framed knots and links. 


\subsection{Chord diagrams and weight systems.}

Definition 1.2.1. A chord diagram of order $n$ is an oriented circle with $n$ non-intersecting pairs of points (chords) on it, up to an orientation preserving diffeomorphism of the circle.

Denote by $\mathcal{D}_{n}$ the set of all chord diagrams of order $n$. There exists a natural forgetting of knottedness map $c h: \mathcal{K}_{n} \rightarrow \mathcal{D}_{n}$ : for every singular knot $K$ with $n$ double points, $\operatorname{ch}(K)$ is the chord diagram order $n$, where the chords are the inverse images of double points under the map $K$ : $S^{1} \rightarrow \mathbb{R}^{3}$.

Drawing chord diagrams we assume that the circle is oriented counterclockwise. The intersection points of chords on these drawings are irrelevant. The only thing that matters is the way the chords combine their endpoints into pairs.

An immediate corollary from the definition of Vassiliev invariants is that the value of an invariant of order $\leq n$ on a singular knot $K$ with $n$ self-intersections depends only on the diagram $\operatorname{ch}(K)$ of $K$, not on its knottedness (i.e. on combinatorics rather than topology of $K$ ).

Proposition 1.2.2. If $I \in V_{n}$ and $K_{1}, K_{2} \in \mathcal{K}_{n}$ such that $\operatorname{ch}\left(K_{1}\right)=$ $\operatorname{ch}\left(K_{2}\right)$, then $I\left(K_{1}\right)=I\left(K_{2}\right)$.

In other words, $I$ descends to a function $C(I)$ on $\mathcal{D}_{n}$.

1.2.3. Vassiliev-Birman-Lin relations for chord diagrams. The properties (1) and (2) of 1.1.2 translate into the following relations between restrictions of $I \in V_{n}$ onto $\mathcal{D}_{n}[1]$.

(1) Isolated chord relation:

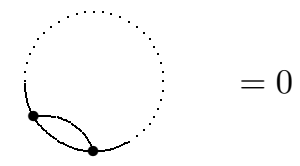

(2) Four-term relation for chord diagrams:

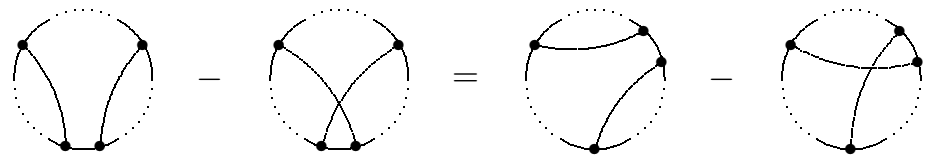

(There may be other chords attached to dotted arcs on these drawings, but there are no other points on the solid arcs.)

\subsection{Kontsevich-Bar-Natan's theorem.}

Definition 1.3.1. A function $W: \mathcal{D}_{p} \rightarrow k$ satisfying the conditions (1) and (2) of 1.2.3 is called a weight system of order $p$. If $W$ satisfies only the 4-term relation (2), then we call it a weak weight system.

Denote by $W_{p}$ the set of all weight systems of order $p$, and by $\widehat{W}_{p}$ the set of all weak systems of order $p$. 
The conditions 1.2.3 imply that there is a natural map $V_{p} / V_{p-1} \rightarrow W_{p}$. It is easy to see that this is a monomorphism: if $I_{1}, I_{2} \in V_{p}$ and $C\left(I_{1}\right)=$ $C\left(I_{2}\right)$, then $I_{1}-I_{2} \in V_{p-1}$. The remarkable fact is that this map is also an epimorphism (at least in the case when $k$ is a field of characteristic zero). In other words, each weight system of order $p$ is a restriction to $\mathcal{D}_{p}$ of some Vassiliev invariant. Let $\mathcal{A}_{p}$ (resp. $\widehat{\mathcal{A}}_{p}$ ) be the space generated by $\mathcal{D}_{p}$ modulo relations (1) and (2) (resp. (2) only), and

$$
\mathcal{A}=\oplus_{p} \mathcal{A}_{p}, \quad \widehat{\mathcal{A}}=\oplus_{p} \widehat{\mathcal{A}}_{p} .
$$

Denote by $\widehat{V}_{p}$ the space of Vassiliev invariants of order $\leq p$ of framed knots.

Theorem 1.3.2. $[3,5]$

(1) $V_{p} / V_{p-1} \simeq W_{p} \simeq \mathcal{A}_{p}^{*}$.

(2) $\widehat{V}_{p} / \widehat{V}_{p-1} \simeq \widehat{W}_{p} \simeq \widehat{\mathcal{A}}_{p}^{*}$.

(3) Operation of connected sum of diagrams induces on $\mathcal{A}$ and $\widehat{\mathcal{A}}$ structures of commutative graded $k$-algebras. The comultiplication dual to this product makes the graded algebras $\mathcal{W}=\oplus_{p} W_{p} \simeq \mathcal{A}^{*}$ and $\widehat{\mathcal{W}}=\oplus_{p} \widehat{W_{p}} \simeq \widehat{\mathcal{A}}^{*}$ commutative and co-commutative Hopf algebras.

(4) $\widehat{\mathcal{A}}=\mathcal{A}[\Theta]$, where $\Theta$ is the primitive element in $\widehat{A}_{1}$ represented by the only chord diagram of order 1 , i.e.

$$
\widehat{\mathcal{A}}_{p}=\mathcal{A}_{0} \otimes \Theta^{p}+\mathcal{A}_{1} \otimes \Theta^{p-1}+\ldots \mathcal{A}_{p} \otimes 1 .
$$

Part (4) of the theorem shows that there is a canonical projection $\widehat{W}_{p} \rightarrow$ $W_{p}$. Therefore, to get a weight system of order $p$, it is enough to construct a function on $\mathcal{D}_{p}$ satisfying only four-term relations. We will not consider one-term relations in the rest of the paper.

\section{Feynman diagrams and graphs}

Now we want to find a way to construct (weak) weight systems, that is to assign a number to each chord diagram such that these numbers satisfy the four-term relations. It seems natural to use the idea of locality that underlies all recent applications of physics to low-dimensional topology (and, in the case of knots and links, is realized in the usage of tangles in works of Reshetikhin, Turaev, Yetter, e.a.). But this idea does not apply immediately to our situation since the four-term relations are not local. A natural way out is to replace the set of chord diagrams by a larger set that allows local relations. Such extension exists and these objects are called Feynman diagrams. 
2.1. Feynman diagrams. The three-term relation on the level of chord diagrams can be drawn as

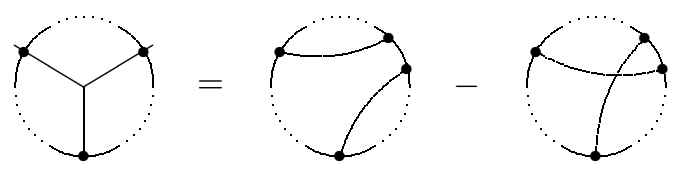

This, in turn, leads to the following generalization of the notion of chord diagram [2].

Definition 2.1.1. A Feynman diagram of order $p$ is a graph with $2 p$ vertices of degrees 1 or 3 with cyclic orders on the set of its univalent (external) vertices and each set of 3 edges meeting at a trivalent (internal) vertex.

We draw Feynman diagrams by placing their external vertices on a circle (Wilson line) with the counterclockwise orientation. We assume that the edges meeting at each internal vertex are also oriented counterclockwise.

Denote by $\mathcal{F}_{p}$ the set of all Feynman diagrams with $2 p$ vertices (up to the natural equivalence of graphs with orientations). The set $\mathcal{D}_{p}$ of chord diagrams with $p$ chords is a subset of $\mathcal{F}_{p}$. Here is an example of a Feynman diagram from $\mathcal{F}_{4}$

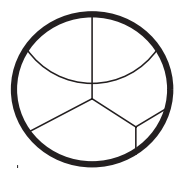

Definition 2.1.2. Denote by $\mathcal{B}_{p}$ the vector space generated by Feynman diagrams of order $p$ modulo relations

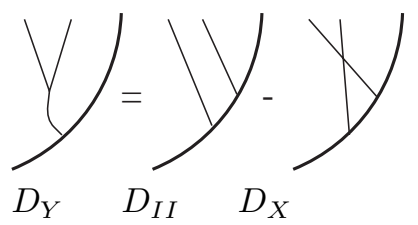

More precisely, $\mathcal{B}_{p}=<\mathcal{F}_{p}>/<D_{Y}-D_{I I}-D_{X}>$, where diagrams $D_{I I}$ and $D_{X}$ are obtained from the diagram $D_{Y}$ by replacing its $Y$-fragment by the $I I$ - and $X$ - fragments respectively. This gives another description of the space $\left(\widehat{V}_{p} / \widehat{V}_{p-1}\right)^{*}=\widehat{\mathcal{A}}_{p}$.

Proposition 2.1.3. [3]

(1) $\mathcal{B}_{p} \simeq \mathcal{A}_{p}$.

(2) The following local relations hold for internal vertices in Feynman diagrams: 

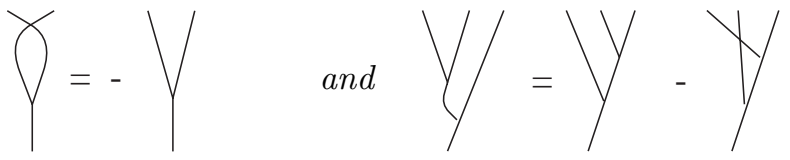

2.2. Feynman graphs: the idea of locality. By analogy with the role played by tangles for knots and links (cf. $[11,16,8]$ ), we introduce Feynman graphs as objects that can be sewn to produce Feynman diagrams.

A Feynman graph is a graph with 1- and 3-valent vertices such that the set of its univalent vertices is a disjoint union of two linearly ordered sets: incoming and outgoing vertices; and the edges meeting at each of its trivalent (internal) vertex have a cyclic order.

We denote by $\mathcal{F}_{a, b}$ the set of Feynman graphs with $a$ incoming and $b$ outgoing vertices and by $\mathcal{F}_{*}$ the set of all Feynman graphs.

We draw Feynman graphs by putting all the incoming vertices on a horizontal line, outgoing vertices on a parallel line below, and all the internal vertices between these two lines (with the counterclockwise orientation at each vertex). For example, the following graph is in $\mathcal{F}_{4,2}$

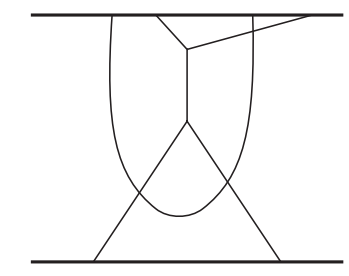

2.2.1. Algebraic operations on $\mathcal{F}_{*}$. There are two natural operations on $\mathcal{F}_{*}$ : composition and tensor product. If $A \in \mathcal{F}_{b, c}$ and $B \in \mathcal{F}_{a, b}$, then their composition $A \circ B \in \mathcal{F}_{a, c}$ is the Feynman graph obtained by attaching outgoing vertices of $B$ to the corresponding incoming vertices of $A$.

The tensor product of $A \in \mathcal{F}_{a, b}$ and $C \in \mathcal{F}_{c, d}$ is the graph $A \otimes C \in$ $\mathcal{F}_{a+c, b+d}$ obtained by placing $C$ to the right of $A$.

For example, if $A=$
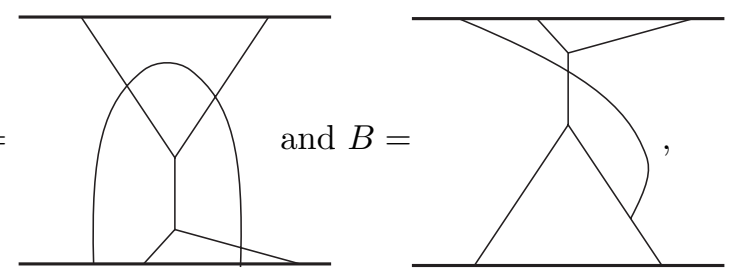

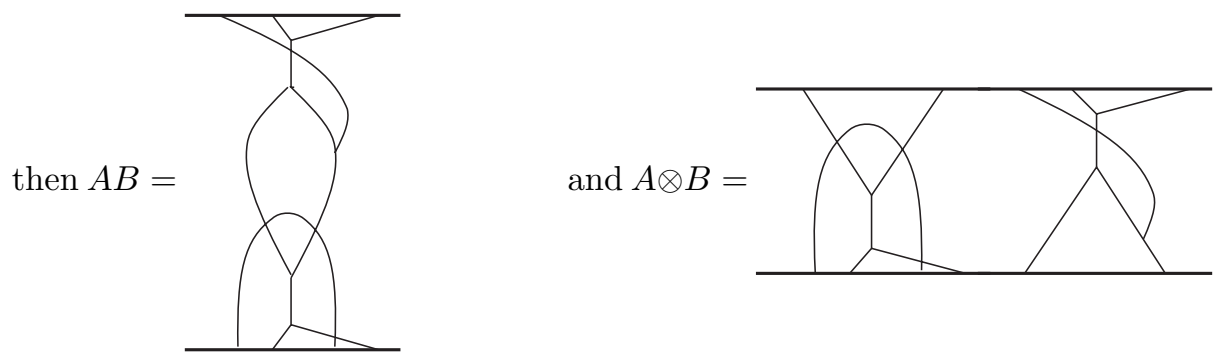

Remark 2.2.2. These operations become "true" compositions and tensor products if we view elements of $\mathcal{F}_{*}$ as morphisms in the category $\mathbf{F G}$ with objects $0,1,2, \ldots$. With respect to these operations, FG is a symmetric monoidal category (cf. [7]).

2.2.3. Generators of $\mathcal{F}_{*}$. With respect to the two operations, $\mathcal{F}_{*}$ is generated by the following elementary graphs:

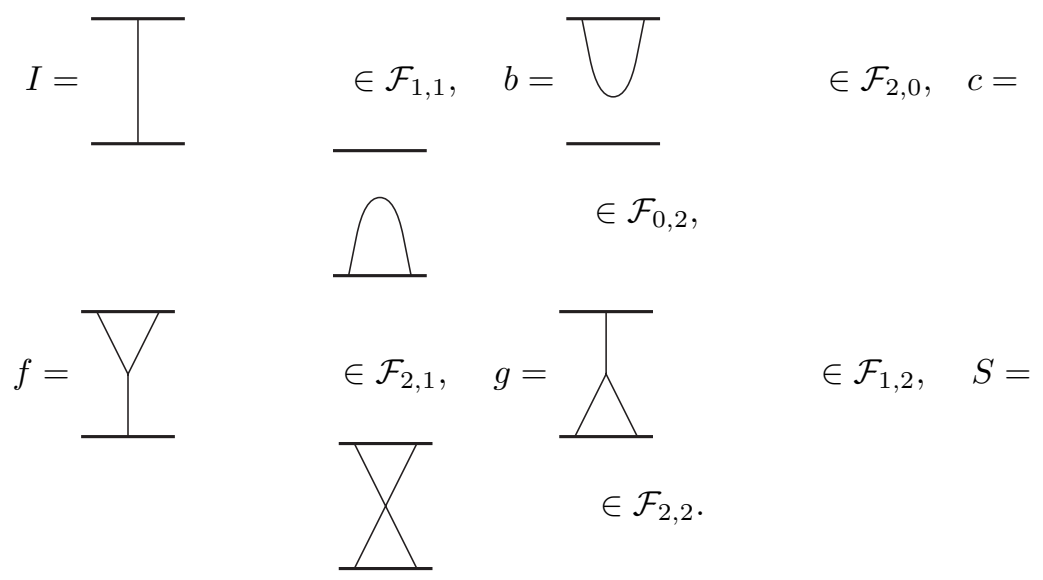

Now we have to describe relations between these generators. 
Theorem 2.2.4. The algebra $\mathcal{F}_{*}=\operatorname{Mor}(\mathbf{F G})$ is generated by the elementary graphs and the following defining relations

(1) $I=i d$

(2) $S^{2}=I \otimes I$<smiles>CCCCC(C)(C)C(C)(C)C(C)C</smiles>

(3) $b S=b$<smiles>CCCC1CCCCC1(C)C</smiles>

(4) $(S \otimes I)(I \otimes S)(S \otimes I)=(I \otimes S)(S \otimes I)(I \otimes S)$ or the quantum Yang-Baxter equation,

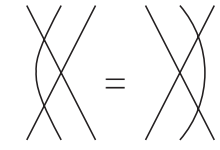

(5) $S(f \otimes I)=(I \otimes f)(S \otimes I)(I \otimes S)$ or

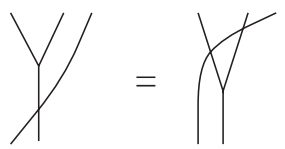

(6) $(I \otimes b)(c \otimes I)=I=(b \otimes I)(I \otimes c)$ or

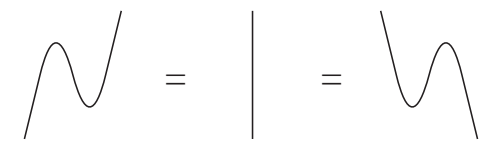

(7) $g=(I \otimes f)(c \otimes I)$ or $\lambda=\bigwedge$

(8) $(b \otimes I)(I \otimes S)=(I \otimes b)(S \otimes I)$ or

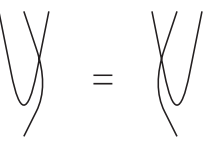

(9) $b(f \otimes I)=b(I \otimes f)$ or

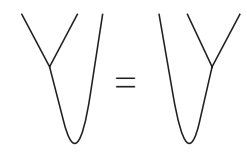

(See [11] for the precise definition of generators and relations in monoidal categories.)

It is clear that $\mathcal{F}_{*}$ is generated by the elementary graphs and that the relations 1-9 hold true in it. The proof that no extra relations are needed is similar to the proofs of the corresponding Reidemeister type results for categories of tangles $[8,11,16]$. 
2.2.5. Category of pictures. Theorem 2.2 .4 asserts that two syntactically correct words in elementary graphs are equal if and only if they can be transformed to each other by a sequence of transformations 2-9 and their inverses. Another way of expressing this is to consider "pictures" - flat graphs with four types of vertices: univalent vertices (incoming and outgoing vertices of Feynman graphs); bivalent vertices (maxima and minima of the edges); 3-valent vertices (internal vertices of Feynman graphs); and 4 -valent vertices (intersection points of edges). All univalent vertices of a picture belong to two parallel horizontal lines, and the rest of the picture is located strictly between these lines. We assume that the edges of the picture do not have horizontal tangents other than at bivalent vertices; that each trivalent vertex has at least one descending and one ascending edge; and that at each four-valent vertex exactly two edges go up and two edges go down. We also assume that all the interior vertices of the picture have different height.

It is clear that each picture corresponds to a Feynman graph and that each Feynman graph can be represented by a picture. Thus the pictures are analogous to knot diagrams for Feynman graphs. The above relations play the role of the Reidemeister moves in this situation.

\section{Operator invariants of Feynman graphs}

3.1. Yang-Baxter algebras. By analogy with the case of tangles and links, let us fix a vector space $L$ and try to assign an operator $I(\Gamma): L^{\otimes a} \rightarrow$ $L^{\otimes b}$ to each Feynman graph $\Gamma \in \mathcal{F}_{a, b}$, such that the map $\Gamma \mapsto I(\Gamma)$ respects composition and tensor product in $\mathcal{F}_{*}$. In other words, we are trying to construct a representation of the monoidal category FG in the monoidal category $\mathcal{T}(L)$ of the tensor powers of $L$.

Since $\mathcal{F}_{*}$ is generated by elementary graphs, a representation of $\mathbf{F G}$ is uniquely specified by choice of a vector space $L$ and a set of homomorphisms (operators):

$$
\begin{aligned}
& b: L \otimes L \rightarrow k \\
& c: k \rightarrow L \otimes L \\
& f: L \otimes L \rightarrow L \\
& g: L \rightarrow L \otimes L \\
& S: L \otimes L \rightarrow L \otimes L
\end{aligned}
$$

By rewriting the relations 2.2.4 (1) - (9) in terms of operators $b, c, f, g, S$, we can find out when this data gives a representation of the category FG. 
Proposition 3.1.1. A set of operators $b, c, f, g, S$ gives a representation of the category $\mathbf{F G}$ in the vector space $L$ if and only if it satisfies the following conditions:

(1) The operator $S$ is a symmetry, i.e. $S^{2}=i d_{L \otimes L}$.

(2) $S$ satisfies the quantum Yang-Baxter equation:

$$
S_{12} S_{23} S_{12}=S_{23} S_{12} S_{23} .
$$

(3) Multiplication $f$ is compatible with symmetry $S: S f=f_{23} S_{12} S_{23}$.

(4) Bilinear form $b$ is $S$-symmetric: $b S=S$.

(5) $b$ is compatible with $S: b_{12} S_{23}=b_{23} S_{12}$.

(6) $b_{23} c_{12}=i d_{L}=b_{12} c_{23}$, i.e. $b$ is a non-degenerate bilinear form and $c$ is its inverse.

(7) Comultiplication $g$ is a conjugate of the multiplication: $g=c_{12} f_{23}$.

(Here we used the standard notation: if $T \in \operatorname{Hom}(V \otimes V, W)$, then $T_{12}$ denotes the operator $T \otimes i d: V \otimes V \otimes U \rightarrow W \otimes U$, etc.)

In particular, relations 6 and 7 show that we do not have any freedom in choosing $c$ or $g$. Rephrasing this Proposition, we see that representations of FG are described by the following algebraic objects.

Definition 3.1.2. A vector space $L$ with an operator $S: L \otimes L \rightarrow L \otimes L$ and a multiplication $f: L \otimes L \rightarrow L$ satisfying conditions 3.1.1.(1-3) is called a Yang-Baxter algebra or, simply, an $S$-algebra. If, in addition, $L$ is finite-dimensional and is equipped with a non-degenerate inner product $b: L \otimes L \rightarrow k$ satisfying 3.1.1.(4-5), it is called a Euclidean $S$-algebra.

Therefore, every Euclidean $S$-algebra $L$ gives a representation of the category FG.

3.2. Yang-Baxter Lie algebras. Now, when can these operator-valued invariants of Feynman graphs be used to produce weight systems (and ultimately, Vassiliev invariants)?

Denote by $\mathcal{G}_{a, b}$ the quotient space of the space $\left\langle\mathcal{F}_{a, b}>\right.$ of formal linear combinations of elements of $\mathcal{F}_{a, b}$ by the three-term and skew-commutativity relations 2.1.3.(2) for internal vertices.

It is clear that $\mathcal{F}_{*}$ induces on $\mathcal{G}_{*}=\bigcup \mathcal{G}_{a, b}$ a structure of a monoidal category with operations extended by linearity. To obtain a representation of the category $\mathcal{G}_{*}$, we have to reformulate conditions 2.1.3.(2) in terms of the tensors $S, f$ and $b$ from 3.1.2. Thus, we come to the following notions.

Definition 3.2.1. A Yang-Baxter Lie algebra is an $S$-algebra $L$, such that the multiplication $f: L \otimes L \rightarrow L$ is $S$-skew-commutative $f \circ S=-f$ and satisfies the $S$-Jacobi identity $f \circ f_{12}=f \circ f_{23}-f \circ f_{23} \circ S_{12}$.

A Euclidean $S$-algebra $L$ with a bilinear form $b$ is called a Euclidean Yang-Baxter Lie algebra if $b$ is $f$-invariant, i.e. $b \circ f_{12}=b \circ f_{23}$. 
Summing up, we have established a one-to-one correspondence between representations of the category $\mathcal{G}_{*}$ and Euclidean Yang-Baxter Lie algebras.

3.3. Weight systems from Yang-Baxter Lie algebras. Given a Euclidean Yang-Baxter Lie algebra $L$, for each Feynman graph $\Gamma \in \mathcal{F}_{0, n}$ we constructed a tensor $I(\Gamma) \in L^{\otimes n}$. To be able to obtain a weight system we have to take into account the relations 2.1.2 for the exterior vertices of Feynman diagrams.

Translating this relation into our tensor notation, we obtain the relation

$$
f(a, b)=a \otimes b-S(a \otimes b)
$$

on the tensor algebra $T^{*}(L)$. Thus, we come to the following notion.

Definition 3.3.1. The universal enveloping algebra $\mathcal{U}_{S}(L)$ of the $S$-Lie algebra $L$ is the quotient algebra of the tensor algebra $T^{*}(L)$ by the ideal generated by the expressions $a \otimes b-S(a \otimes b)-f(a, b)$ for $a, b \in L$.

Universal enveloping algebras of $S$-Lie algebras are completely analogous to universal enveloping algebras of ordinary Lie algebras. In particular, there is a version of the Poincaré-Birkhoff-Witt theorem for them and a natural action of $L$ on $\mathcal{U}_{S}(L)$.

Thus, we have a homomorphism $W_{L}$ from the algebra $\mathcal{G}_{*}$ to $\mathcal{U}_{S}(L)$. The following fact makes it possible to pull $W_{L}$ down to the space of Feynman diagrams modulo 3 -term relations.

Proposition 3.3.2. The elements $W_{L}(\Gamma) \in \mathcal{U}_{S}(L)$ are invariant with respect to the canonical $L$ action. (In other words, they belong to the $S$ center of $\mathcal{U}_{S}(L)$.)

Summing up, we arrive to our main result.

\section{Theorem 3.3.3.}

(1) For every Euclidean Yang-Baxter Lie algebra $L$ there exists a natural homomorphism of algebras $\rho_{L}: \widehat{\mathcal{A}} \rightarrow \mathcal{U}_{S}(L)$.

(2) The image of $\rho_{L}$ belongs to the $S$-center of $\mathcal{U}_{S}(L)$.

(3) Every linear functional $\phi$ on the center $Z_{S}\left(\mathcal{U}_{S}(L)\right)$ provides sequences of weight systems $\hat{\phi}_{n}: \widehat{\mathcal{A}}_{n} \rightarrow k$ and $\phi_{n}: \mathcal{A}_{n} \rightarrow k$. The sequence $\phi_{n}$ is non-trivial if $L$ is not $S$-commutative.

\section{Concluding remarks and examples}

4.1. Lie algebras. If the symmetry operator $S$ is the standard permutation $S(u \otimes v)=v \otimes u$, then $S$-Lie algebras are just Lie algebras. In this case our construction of Vassiliev weight systems becomes Bar-Natan's construction [2].

A source of central functions on $\mathcal{U}(L)$ in this case is provided by traces $\operatorname{tr}(\rho(a))$, where $a \in \mathcal{U}(L)$ and $\rho$ is a finite-dimensional representation of 
$L$. If $L$ is a semisimple (or reductive) Lie algebra over $\mathbf{C}$, then all central functions are linear combinations of traces in irreducible representations. As it is explained in [3], the corresponding knot invariants of a knot and of the same knot with the opposite orientation always coincide. Therefore, if Vassiliev's completeness conjecture is correct, there should be weight systems which are not linear combinations of weight systems coming from semisimple Lie algebras.

4.2. Modules and $S$-traces. To generalize the construction of weight systems using representations of Lie algebras, we need to define corresponding notions for $S$-Lie algebras.

The three-term relation in 2.1.2 gives us the definition of a module.

Definition 4.2.1. A module over $S$-Lie algebra $L$ is a vector space $M$ with a homomorphism $m: L \otimes M \rightarrow M$ such that

$$
m\left(f \otimes \operatorname{id}_{M}\right)=m\left(\mathrm{id}_{L} \otimes m\right)-m\left(\operatorname{id}_{L} \otimes m\right)\left(S \otimes \operatorname{id}_{M}\right) .
$$

As usual, modules over $S$-Lie algebra $L$ are the same as modules over the associative algebra $\mathcal{U}_{S}(L)$.

In order to produce invariant functionals on $\mathcal{U}_{S}(L)$ we need one more definition.

Definition 4.2.2. Let $M$ be a module over $S$-Lie algebra $L$. A functional $\phi \in \operatorname{Hom}(M, M)^{*}$ is called an $S$-trace on $M$ if $\phi(m(f(a, b)))=0$ for all $a, b \in L$. (Here $f$ is the multiplication on $L$ and $m$ is considered as homomorphism $L \rightarrow \operatorname{Hom}(M, M)$.)

There is no trace on a module a priori, but usually modules come equipped with one.

4.3. Lie superalgebras. Let $L=L_{\overline{0}} \oplus L_{\overline{1}}$ be a $\mathbb{Z}_{2}$-graded vector space and $S$ be the standard super-permutation

$$
S(u \otimes v)=(-1)^{|u||v|} v \otimes u,
$$

where $u \in L_{|u|}$ and $v \in L_{|v|}$.

Proposition 4.3.1. If $S$ is the super-permutation, then $S$-Lie algebras are Lie superalgebras.

The only non-obvious thing here is that the multiplication $f$ respects the $\mathbb{Z}_{2}$-grading which is required by the definition of Lie superalgebras. It follows from the consistency of $f$ and $S$ (condition 3.1.1.3). Similarly, condition 3.1.1.5 shows that the bilinear form $b$ in a Euclidean Lie superalgebra must be even.

Let $M$ be an $L$-module. In general, there is no $\mathbb{Z}_{2}$-grading on $M$, therefore $M$ is not necessarily a representation of the Lie superalgebra $L$. In the case when $M$ is a Lie superalgebra module (i.e. $M=M_{\overline{0}} \oplus M_{\overline{1}}$ and $L_{a} M_{b} \subset M_{a+b}$ ), there is a natural $S$-trace on $M$ - the supertrace 
$\operatorname{str}(h)=\operatorname{tr}\left(h_{\overline{0}}\right)-\operatorname{tr}\left(h_{\overline{1}}\right)$, where $h \in \operatorname{Hom}(M, M)$ and $h_{a}$ is the composition $M_{a} \rightarrow M \rightarrow M / M_{a+\overline{1}} \simeq M_{a}$.

In the case of Lie superalgebras not all central functionals are linear combinations of supertraces. For example, all supertraces in non-trivial irreducible representations $M$ of the Lie superalgebra $L=\mathcal{G l}(n \mid n)$ vanish on $Z(\mathcal{U}(L))$. But a central element $c \in \mathcal{U}(L)$ acts on $M$ as a scalar operator. Let this operator be $\lambda_{M}(c) i d_{M}$. Then the functional $\lambda_{M}$ gives an example of a central function which is not a combination of supertraces.

In the simplest case when $L=\mathcal{G l}(1 \mid 1)$ and $M$ is its standard (1|1)dimensional module, the sequence of weight systems constructed by 3.3.3 for the functional $\lambda_{M}$ coincides with the sequence of weight systems of coefficients of the Alexander-Conway polynomial.

Even the vanishing supertraces can provide some valuable information. For example, in the standard $(2 n \mid 2 n)$-dimensional representation of the Lie superalgebra $L=\mathcal{O} \mathcal{S} p(2 n \mid 2 n)$, the supertraces of central elements of $\mathcal{U}(L)$ vanish and so do the corresponding weight systems. However, in terms of the even subalgebra $L_{\overline{0}}=\mathcal{O}(2 n) \oplus \mathcal{S} p(2 n) \subset L$, this translates into a relationship between the weight systems given by the standard representations of the Lie algebras $\mathcal{O}(2 n)$ and $\mathcal{S} p(2 n)$. This relationship was found by Bar-Natan [3] by direct computations. (The details will appear in $[12]$.

4.4. Other $S$-Lie algebras. There are many examples of Euclidean Yang-Baxter Lie algebras other than Lie algebras and Lie superalgebras. For each involutive solution $S$ of the quantum Yang-Baxter equation there are several series of Euclidean Yang-Baxter Lie algebras, analogues of the classical $G l, O$ and $S p$ series (cf. [4, 6, 12]). There is no obvious reason to believe that the corresponding Vassiliev invariants belong to the space generated by the invariants coming from Lie algebras. Already in the case of Lie superalgebras, we have more algebras, more representations and more central functionals to work with.

On the other hand, the notion of the Yang-Baxter Lie algebra turned out to be intrinsically related to Vassiliev-Birman-Lin diagrams. This allows us to expect that all Vassiliev invariants can be obtained by working with Yang-Baxter Lie algebras.

4.5. Yang-Baxter Chern-Simons models. Vassiliev invariants coming from Lie algebras appear as coefficients of perturbative expansions of the corresponding Chern-Simons-Witten invariants. One may ask if there exists such a correspondence for Vassiliev invariants coming from YangBaxter Lie algebras.

For each $S$-Lie algebra $L$ we can define principal $L$-bundles over manifolds and develop a formalism of $L$-connections parallel to the standard one. In particular, given an invariant inner product on $L$ we construct an 
analogue of the Chern-Simons 3-form [12]. This may lead to new invariants of 3-manifolds of Reshetikhin-Turaev-Witten type. The relation between the corresponding knot invariants and the Vassiliev invariants constructed from the same data must be similar to the relationship between the two constructions of knot invariants based on ordinary Lie algebras. The work of Rozansky and Saleur [9] found that the Alexander-Conway polynomial is the Chern-Simons-Witten invariant for the standard representation of the Lie superalgebra $\mathcal{G l}(1 \mid 1)$. This matches our computations of the corresponding weight systems and supports this conjecture.

\section{Acknowledgment}

This paper is based on the talks I gave at the conference "Quantum Topology" (April 1993, Manhattan, Kansas) and the AMS special session on Invariants of Low Dimensional Manifolds (April 1994, Brooklyn). I would like to thank the organizers of these meetings for inviting me there and giving me an opportunity to speak. I am grateful to S. Chmutov, S. Duzhin and S. Lando for permission to use in Section 1, drawings from their preprint "Vassiliev knot invariants I. Introduction". I also thank referees for valuable remarks.

\section{References}

[1] J.S. Birman and X.-S. Lin, Knot polynomials and Vassiliev's invariants, Invent. Math. 111 (1993), 225-270.

[2] D. Bar-Natan, Weights of Feynman diagrams and Vassiliev knot invariants, preprint, 1991.

[3] D. Bar-Natan, On the Vassiliev knot invariants, Topology, to appear.

[4] D. Gurevich, The Yang-Baxter equation and a generalization of formal Lie theory, Soviet Math. Dokl. 33 (1986), 758-762.

[5] M. Kontsevich, Vassiliev's knot invariants, Adv. in Soviet Math. 16, Amer. Math. Soc., 1993

[6] V. Lyubashenko, Hopf algebras and vector symmetries, Russian Math. Surveys 41 (1986), 153-154.

[7] S. Mac Lane, Categories for the working mathematician, Springer-Verlag GTM 5, 1971.

[8] N. Reshetikhin, Quasitriangular Hopf algebras and invariants of tangles, Leningrad Math. J. 1 (1990), 491-513.

[9] L. Rozansky, H. Saleur, $S$ and $T$ matrices for the super $U(1,1)$ WZW model. Application to surgery and 3-manifolds invariants based on the Alexander-Conway polynomial, Nucl. Phys. B376 (1992), 461.

[10] V.G. Turaev, The Yang-Baxter equation and invariants of links, Invent. Math. 92 (1988), 527-553.

[11] V.G. Turaev, Operator invariants of tangles and R-matrices, Math. USSR Izvestia 35 (1990), 411-444.

[12] A. Vaintrob, Yang-Baxter models for Vassiliev knot invariants, in preparation.

[13] V.A. Vassiliev, Cohomology of knot spaces, Theory of singularities and its applications (V.I.Arnold, ed.), Amer. Math. Soc., 1990, 23-69. 
[14] V.A. Vassiliev, Complements of discriminants of smooth maps: topology and applications. Transl. of Math. Monogr. 98, Amer. Math. Soc., 1992.

[15] E. Witten, Quantum field theory and Jones polynomial, Commun. Math. Phys. 121 (1989), 351-399.

[16] D.N. Yetter, Markov algebras, Braids, Contemp. Math. 78, Amer. Math. Soc., 1988.

Department of Mathematics, New Mexico State University, Las Cruces, New Mexico 88003

E-mail address: vaintrob@math.nmsu.edu 